$$
\begin{aligned}
& \sum_{j=0}^{d r-q}(-1)^{j}\left(\begin{array}{c}
d r \\
j
\end{array}\right)\left(\left(\begin{array}{c}
d r-j \\
d
\end{array}\right)\right)=(d r) ! /\left[r !(d !)^{r}\right], \\
& \sum_{j=0}^{d r-q-1}(-1)^{j}\left(\begin{array}{c}
d r-1 \\
j
\end{array}\right)\left(\left(\begin{array}{c}
d r-j-1 \\
d \\
r
\end{array}\right)\right)=d(d r) !(r-1) / 2\left[r !(d !)^{r}\right], \text { etc. }
\end{aligned}
$$

Details of proofs, computations, and applications will appear elsewhere.

\title{
REFERENCES
}

1. Z. A. Melzak, Scattering from random arrays, Quart. Appl. Math. 20 (1962), 151-159.

2. J. Riordan, Combinatorial analysis, Wiley, New York, 1958.

3. G. Uhlenbeck and G. W. Ford, Lectures in statistical mechanics, Lectures in Applied Math., Vol. I, Amer. Math. Soc., Providence, R. I., 1963.

Bell Telephone laboratories, Inc., Murray Hill, New Jersey

\section{FIXED POINTS OF NONEXPANDING MAPS}

\section{BY BEN JAMIN HALPERN}

Communicated by F. Browder, July 12, 1967

Introduction. This paper is concerned with nonexpanding maps from the unit ball of a real Hilbert space into itself. Browder [1] has established that such maps always possess at least one fixed point. We shall develop a method, which resembles the simple iterative method, for approximating fixed points of such maps. In fact, we shall generate a sequence, $\left\{x_{n}\right\}$, by the recursive formula $x_{n+1}$ $=k_{n+1} f\left(x_{n}\right)$ where $f$ is the map in question and $\left\{k_{n}\right\}$ is a sequence of real numbers. Our main result is Theorem 3 which states sufficient conditions on $k_{n}$ to insure the strong convergence of $x_{n}$ to a fixed point of $f$.

Definitions and preliminary observations. Let $H$ be a Hilbert space with inner product denoted by $($,$) and norm by \|\|$. Let $B$ be the unit ball, $B=\{x \in H \mid\|x\| \leqq 1\}$. A map $f: B \rightarrow B$ is nonexpanding if $\|f(x)-f(y)\| \leqq\|x-y\|$ for all $x, y \in B$.

Assume that $f: B \rightarrow B$ is nonexpanding. It is not difficult to establish that the set $F$ of fixed points must be convex. Using the con- 
vexity of $F$ it is then easy to see that there is at most one $y \in F$ such that $\|y\|=\inf _{z \in F}\|z\|$. Now if $g: B \rightarrow B$ is defined by $g(x)=k f(x)$ with $|k|<1$ then $g$ satisfies $\|g(x)-g(y)\| \leqq|k|\|x-y\|$ for all $x, y \in B$. Consequently there exists a unique point $y_{k} \in B$ such that $y_{k}=g\left(y_{k}\right)$ $=k f\left(y_{k}\right)$.

We begin by giving a new proof of a result of Browder [2].

THEOREM 1. Let $f: B \rightarrow B$ be a nonexpanding map and $y_{k}$ the unique element of $B$ satisfying $y_{k}=k f\left(y_{k}\right)$ for $|k|<1$. Then

$$
\lim _{k \rightarrow 1 ;|k|<1} y_{k}=y
$$

where $y$ is the unique fixed point of $f$ with the smallest norm.

Proof. It is obviously sufficient to show that $y_{k_{i}} \rightarrow y$ as $i \rightarrow \infty$ for each sequence $k_{i}, i=1,2, \cdots$, satisfying $k_{1}<k_{2}<k_{3}<\cdots$ and $k_{i} \rightarrow 1$ as $i \rightarrow \infty$. The existence of $y$ will be established in the course of the proof.

Assume that $0<k<l \leqq 1, y_{k}=k f\left(y_{k}\right), y_{l}=l f\left(y_{l}\right)$ and $d=y_{l}-y_{k}$. Then using $\left\|f\left(y_{l}\right)-f\left(y_{k}\right)\right\| \leqq\left\|y_{l}-y_{k}\right\|$ we obtain

$$
\left(l^{-1}\left(y_{k}+d\right)-k^{-1} y_{k}, l^{-1}\left(y_{k}+d\right)-k^{-1} y_{k}\right) \leqq\|d\|^{2} .
$$

Thus

$$
\left(l^{-1}-k^{-1}\right)^{2}\left\|y_{k}\right\|^{2}+\left(l^{-2}-1\right)\|d\|^{2} \leqq 2\left(k^{-1}-l^{-1}\right) l^{-1}\left(y_{k}, d\right) .
$$

Consequently, $\left(y_{k}, d\right) \geqq 0$.

Now note

$$
\left\|y_{l}\right\|^{2}=\left(y_{k}+d, y_{k}+d\right)=\left\|y_{k}\right\|^{2}+\|d\|^{2}+2\left(y_{k}, d\right)
$$

and so

$$
\left\|y_{l}\right\|^{2} \geqq\left\|y_{k}\right\|^{2}+\left\|y_{l}-y_{k}\right\|^{2} .
$$

The sequence $\left\|y_{l}\right\|$, being monotonic and bounded, converges. Hence $\left\|y_{l}-y_{k}\right\| \leqq\left\|y_{l}\right\|{ }^{2}-\left\|y_{k}\right\|^{2} \rightarrow 0$ as $k, l \rightarrow+\infty$. Thus $y_{k_{i}}$ converges to some $q$ as $i \rightarrow \infty$. Since $B$ is closed $q \in B$. Note that because $f$ is nonexpanding it is continuous. Thus if we take the limit of both sides of (3) below as $i \rightarrow \infty$ we find that $q$ is a fixed point of $f$.

$$
y_{k_{i}}=k_{i} f\left(y_{k_{i}}\right) \text {. }
$$

Now let $p$ be any fixed point of $f$. Then $p=1(f(p))$ and so (2) holds with $p=y_{l}, l=1, y_{k_{i}}=y_{k}$ and $k=k_{i}$ for any $i=1,2, \cdots$. Since $y_{k} \rightarrow q$ as $i \rightarrow \infty,\left\|y_{k_{i}}\right\| \rightarrow\|q\|$ as $i \rightarrow \infty$ and thus $\|q\| \leqq\|p\|$. Therefore $\|q\|$ $=\inf \|p\|, p$ a fixed point of $f$. As we have noted above there can be no 
more than one such fixed point of $f$, which we call $y$. We have thus shown that $y_{k_{i}} \rightarrow y$ as $i \rightarrow \infty$ for each sequence $k_{i}, i=1,2, \cdots$ satisfying $k_{1}<k_{2}<k_{3}<\cdots$ and $k_{i} \rightarrow 1$ as $i \rightarrow \infty$. This proves the theorem.

Principal results. We know that if we pick any $x_{0} \in B$ and define $x_{n}, n=1,2, \cdots$, inductively by the formula $x_{n}=k f\left(x_{n-1}\right)$ with $|k|<1$ then $x_{n} \rightarrow y_{k}$ as $n \rightarrow \infty$. We also know that $y_{k} \rightarrow y$ as $k \rightarrow 1$, $|k|<1$. This suggests the following question. What sequences of real numbers $\left\{k_{i}\right\}, i=1,2, \cdots$, have the property that if we define the sequence $z_{n}$ inductively by

$$
z_{0}=a, \quad z_{n+1}=k_{n+1} f\left(z_{n}\right), \quad n=0,1, \cdots,
$$

and let $y$ be the fixed point of $f$ with the smallest norm, then

$$
z_{n} \rightarrow y \text { as } n \rightarrow \infty
$$

irrespective of our choice of Hilbert space $H$, nonexpanding map $f: B \rightarrow B$ where $B=\{x \mid x \in H,\|x\| \leqq 1\}$, and starting point $a \in B$. Such a sequence $\left\{k_{i}\right\}$ will be called acceptable.

TheOREM 2. Three necessary conditions for $\left\{k_{i}\right\}, i=1,2, \cdots$, to be acceptable are

(i) $\left|k_{i}\right| \leqq 1$, all $i=1,2, \cdots$,

(ii) $k_{i} \rightarrow 1$ as $i \rightarrow \infty$, and

(iii) $\prod_{i=1}^{\infty} k_{i}=0$.

Proof. To establish the necessity of a condition on $\left\{k_{i}\right\}$ we need only consider a particular $H, f$, and $a$. Take $H$ to be the reals and $a=1$. Then if we set $f(x)=1$ for all $x \in B=\{x|| x \mid \leqq 1\}$ we see that $z_{n}=k_{n}$ for $n=1,2, \cdots$. Condition (i) is necessary in order that $z_{n} \in B$. This is required so that $z_{n+1}$ is defined which, in turn, is needed for (5) to make sense. In this example $y=1$ and so $z_{n} \rightarrow y$ implies $k_{n} \rightarrow 1$. This shows that (ii) is necessary.

Now take $H$ and $a$ as before and set $f(x)=-x$ for $x \in B$. Then $y=0$ and $z_{n}=(-1)^{n} \prod_{i=1}^{n} k_{i}$. Therefore $z_{n} \rightarrow y$ implies $\prod_{i=1}^{\infty} k_{i}=0$. This proves that (iii) is necessary and completes the proof.

THEOREM 3. Sufficient conditions for $\left\{k_{i}\right\}$ to be acceptable are

(i) $k_{i}<1$ for $i=1,2, \cdots$,

(ii) $k_{i} \leqq k_{i+1}$ for $i=1,2, \cdots$,

(iii) $k_{i} \rightarrow 1$ as $i \rightarrow \infty$,

(iv) There exists a sequence $n(i)$ such that

(a) $n(i+1) \geqq n(i)$ for $i=1,2, \cdots$,

(b) $\epsilon_{i+n(i)} \epsilon_{i}^{-1} \rightarrow 1$ as $i \rightarrow \infty$,

(c) $n(i) \epsilon_{i} \rightarrow \infty$ as $i \rightarrow \infty$, where $\epsilon_{i}=1-k_{i}$. 
Proor. We let $y_{i}=k_{i} f\left(y_{i}\right)$ as above and observe that if $l<n$

$$
\begin{aligned}
\left\|z_{l+1}-y_{i}\right\| & =\left\|k_{l+1} f\left(z_{l}\right)-k_{i} f\left(y_{i}\right)\right\| \\
& \leqq k_{i}\left\|z_{l}-y_{i}\right\|+\left|k_{l+1}-k_{i}\right| \\
& \leqq k_{i}\left\|z_{l}-y_{i}\right\|+k_{n}-k_{i} .
\end{aligned}
$$

For $m<n$, set $w_{0}=\left\|z_{m}-y_{i}\right\|$ and $w_{j+1}=k_{i} w_{j}+k_{n}-k_{i}$. Then an easy induction shows that

$\left\|z_{m+j}-y_{i}\right\| \leqq w_{j}=k_{i}^{j}\left\|z_{m}-y_{i}\right\|+\left(k_{n}-k_{i}\right)\left(1+k_{i}+k_{i}^{2}+\cdots+k_{i}^{j-1}\right)$.

Noting that

$$
\sum_{l=0}^{+1} k_{i}^{l} \leqq \sum_{l=0}^{\infty} k_{i}^{l}=\left(1-k_{i}\right)^{-1}
$$

we obtain the estimate

(6) $\quad\left\|z_{n}-y_{i}\right\| \leqq k_{i}^{n-m}\left\|z_{m}-y_{i}\right\|+\left(k_{n}-k_{i}\right)\left(1-k_{i}\right)^{-1} \quad(m<n)$.

Next we will show that $\left\|z_{i+n(i)}-y_{i}\right\| \rightarrow 0$ as $i \rightarrow \infty$. Using (6) with $m=i$ and $n=i+n(i)$ we obtain

$$
\left\|z_{i+n(i)}-y_{i}\right\| \leqq 2 k_{i}^{n(i)}+\left(k_{i+n(i)}-k_{i}\right)\left(1-k_{i}\right)^{-1},
$$

i.e.,

$$
\left\|z_{i+n(i)}-y_{i}\right\| \leqq 2 k_{i}^{n(i)}+\left(\epsilon_{i}-\epsilon_{i+n(i)}\right) \epsilon_{i}^{-1} .
$$

By condition (iv)(b) we have

$$
\left(\epsilon_{i}-\epsilon_{i+n(i)}\right) \epsilon_{i}^{-1}=1-\epsilon_{i+n(i)} \epsilon_{i}^{-1} \rightarrow 0 \quad \text { as } i \rightarrow \infty .
$$

Since $\epsilon_{i} \rightarrow 0$ as $i \rightarrow+\infty$,

$$
\log \left(k_{i}^{n_{i}}\right)=n(i) \log \left(1-\epsilon_{i}\right) \rightarrow-\infty \quad \text { as } i \rightarrow \infty
$$

if $n(i) \epsilon_{i} \rightarrow \infty$ as $i \rightarrow \infty$. But this is condition (iv)(c). Therefore

$$
\left\|z_{i+n(i)}-y_{i}\right\| \rightarrow 0 \text { as } i \rightarrow \infty .
$$

-Since $n(i+1) \geqq n(i)$, all $i$, for any $n$ sufficiently large there exists a unique $j$ such that $j+n(j) \leqq n<j+1+n(j+1)$. This $j$ increases without bound as $n \rightarrow \infty$. Now using (6) with $m=j+n(j)$ and $i=j$ +1 we obtain 


$$
\begin{aligned}
\left\|z_{n}-y_{j+1}\right\| \leqq & k_{j+1}^{n-j-n(j)}\left\|z_{j+n(j)}-y_{j+1}\right\|+\left(k_{n}-k_{j+1}\right)\left(1-k_{j+1}\right)^{-1} \\
\leqq & 1\left(\left\|z_{j+n(j)}-y_{j}\right\|+\left\|y_{j}-y\right\|+\left\|y-y_{j+1}\right\|\right) \\
& +\left(\epsilon_{j+1}-\epsilon_{j+1+n(j+1)}\right) \epsilon_{j+1}^{-1} .
\end{aligned}
$$

It now follows from (7), Theorem 1, and condition (iv)(b) that $\left\|z_{n}-y_{j+1}\right\| \rightarrow 0$ as $n \rightarrow \infty$. But $y_{j+1} \rightarrow y$ as $n$ and thus $j$ approaches infinity. Therefore $z_{n} \rightarrow y$ as $n \rightarrow \infty$. Q.E.D.

COROLlaRy. If $k_{i}=1-i^{-x}$ with $0<x<1$, then $\left\{k_{i}\right\}$ is acceptable.

Proof. Pick $r$ such that $x<r<1$. Then set $n(i)=\left[i^{r}\right]$ where $[t]$ is the greatest integer equal to or less than $t$. It is easy to verify that $\left\{k_{i}\right\}$ and $n(i)$ satisfy conditions (i)-(iv) of Theorem 3 once one notes that $n(i) i^{-r} \rightarrow 1$ as $i \rightarrow \infty$.

ThEOREM 4. Let $\left\{k_{i}\right\}$ and $\left\{m_{i}\right\}$ satisfy $\left|k_{i}\right|<1, k_{i} \rightarrow 1$, and $k_{i}{ }^{m_{i}} \rightarrow 0$. If $x_{0} \in B$ and $x_{n}$ are defined inductively by

$$
x_{n}=k_{n} f\left(k_{n} f \cdots\left(k_{n} f\left(x_{n-1}\right)\right) \cdots\right) \quad n=1,2, \cdots m_{n} \text { times, }
$$

then $x_{n} \rightarrow y$ as $n \rightarrow \infty$.

Proof. An easy induction on the following inequality (8) for $q \in B$

$$
\left\|k_{n} f(q)-y_{k_{n}}\right\|=\left\|k_{n} f(q)-k_{n} f\left(y_{k_{n}}\right)\right\| \leqq k_{n}\left\|q-y_{k_{n}}\right\|
$$

yields

$$
\left\|x_{n}-y_{k_{n}}\right\| \leqq k_{n}^{m_{n}}\left\|x_{n-1}-y_{k_{n}}\right\| \leqq 2 k_{n}^{m_{n}} \text {. }
$$

Thus $\left\|x_{n}-y_{k_{n}}\right\| \rightarrow 0$ as $n \rightarrow \infty$ and since $y_{k_{n}} \rightarrow y$ as $n \rightarrow \infty, x_{n} \rightarrow y$ as $n \rightarrow \infty$. Q.E.D.

\section{REFERENCES}

1. F. E. Browder, Fixed-point theorems for noncompact mappings in Hilbert space, Proc. Nat. Acad. Sci. U.S.A. 53 (1965), 1272-1276.

2. - Convergence of approximants to fixed points of non-expansive nonlinear maps in Banach spaces, Arch. Rational Mech. Anal. 24 (1967), 82-90.

University of California, Berkeley 